\title{
An Experimental Study of the Effect of Load and Moving Speed on Free Rotating Rubber Contact using Fluorescence Microscopy
}

\author{
Jhonni Rahman ${ }^{1 *}$, Yutaka Shoukaku², Tomoaki Iwai ${ }^{2}$ \\ ${ }^{1}$ Department of Mechanical Engineering, Faculty of Engineering, Universitas Islam Riau, Jl. Kaharuddin Nst. \\ No. 113, Simpang Tiga, Kec. Bukit Raya, Kota Pekanbaru 28284, Riau, Indonesia \\ ${ }^{2}$ Division of Mechanical Science and Engineering, Graduate School of Natural Science and Technology, \\ Kanazawa University, Kakuma-machi, Kanazawa 920-1192, Ishikawa, Japan
}

\begin{abstract}
An experimental study was conducted to evaluate the effect of the applied load and moving speed on the free rotating rubber specimen contact area on a smooth-surfaced glass plate. The contact area of the rubber specimen and flat surface was observed using fluorescence microscopy by utilizing ultraviolet as an excitation light source and pyranine as a dye substance. The apparent contact area between the rubber specimen and the flat surface was measured using image processing software based on the Otsu thresholding method. The result reveals an increasing trend line due to applied normal load dependency, which agreed with the Hertz theory. On movingspeed influence, the trend line of rubber contact increased at a lower speed, reaching the highest value at a moving speed of $8 \mathrm{~mm} / \mathrm{s}$, and decreased as the moving speed increased further.
\end{abstract}

Keywords: Apparent contact area; Fluorescence; Free rotating; Rubber

\section{Introduction}

Since the past few decades, many scientists have been conducting several experiments related to the contact mechanics of soft materials due to their significant applications in many industries (Nakajima and Takahashi, 2002; Fujii, 2008; Bódai and Goda, 2012; Fowell et al., 2014). One of them is the research about rubber tires of vehicles. The important role of a tire in the vehicle system can be comprehended as its function in transmitting forces on the vehicle through tire rubber to the road surface in a safe and comfortable manner. Therefore, traction force or grip between the tire and road surface becomes one of the most important performance characteristics needed to understand in the automotive industry (Liang et al., 2020). For example, it helps us to understand what range of friction is needed to keep the vehicles safe when braking or cornering or how fast the vehicle can be utilized to maintain the vehicle under control. If the frictional characteristics fail to satisfy the required tractional force, then the entire system of the vehicle will be out of control, leading to a car accident.

In general, the grip performance of rubber tires is affected by both internal and external factors. One internal factor is the filler inside the tire, which greatly affects its performance. Thus, enhancing the quality of the filler enables the tire to increase its grip performance.

*Corresponding author's email: jhonni_rahman@eng.uir.ac.id, Tel.: +81-80-8049-0623 doi: 10.14716/ijtech.v12i4.4674 
This result was reported by Hasan et al. (2020) in their study about the properties of tire rubber by modifying the clay filler of tires.

Similarly, external factors such as moving speed and loads on vehicles also have a significant impact on vehicle performance. The more weight is loaded on the vehicle, the faster the tire wears down. Faster vehicle runs cause the driver to lose control easily. Therefore, studying these two factors is vital to maintain the tire life and also for driving safety. Although the importance of tire grip performance is applied on both dry and wet surface conditions, its influence is much more reduced on wet surfaces. This phenomenon was shown in a report from Micheline Corporation (Michelin, 2001). Zheng et al. also reported similar results, as the adhesion coefficient reduced when the tire ran on a wet surface, and it reduced further as the water thickness increased (Zheng et al., 2018). Due to its significant impact on the grip performance of tires, we decided to observe and measure the contact condition of tire rubber on wet surfaces.

Tire grip performance or the coefficient of friction is generally calculated based on frictional force, as stated in "laws of friction." It was reported that the friction force $\left(F_{\mu}\right)$ of elastomer contact is generally produced by two types of forces: adhesion force $\left(F_{a}\right)$ and hysteresis force $\left(F_{h}\right)$ (Tabor, 1960). Thus, frictional force can be expressed as follows:

$$
F_{\mu}=F_{a}+F_{h}
$$

However, in rough surface contacts such as between the tire and road surface, the most dominant factor on the friction force is hysteresis force; in contrast, the effect of adhesion force is very minimal (Persson, 2001). On the other hand, when the surface of rubber contact is very smooth, the adhesion force makes the biggest contribution to the friction force (Persson and Volokitin, 2006). As the current study utilized a smooth surface of both the rubber wheel and glass plate, the frictional force solely depends on the adhesion force. Thus, the coefficient of friction can be expressed as follows:

$$
F_{\mu}=F_{a}
$$

Maegawa et al. (2015) reported that the correlation between kinetic friction force and the contact area of the elastomer was linear. Therefore, the frictional force of the elastomer can also be measured based on the contact area between the rubber tire and the mating surface. The coefficient of friction of adhesion force can be expressed as follows:

$$
F_{\mu}=F_{a}=\tau A
$$

where $\tau$ is the shear strength and $A$ is the contact area between the elastomer and the mating surface. As the shear strength does not change, the only factor affecting the adhesion force is the elastomer contact area. This means that the contact area of tire rubber determines the amount of frictional force of the vehicles in a certain manner.

The only way to directly observe the contact condition in situ is by using visualization methods such as fluorescence microscopy. Fluorescence microscopy is the best method for observing and measuring the contact area of elastomer material. Fluorescence microscopy provides detailed information based on the amount of fluorescence dye that exists within the contacting parts. Therefore, the intensity produced in fluorescence microscopy represents the gap within mating contact. Moreover, differentiating the contact region and noncontact region is becoming easier and more accurate. There are several samples of fluorescence microscopy applications in the field of tribology. For example, Fowell et al. (2014) used fluorescence microscopy on elastomeric seal material to measure the lubricant film thickness of contact area at several entrainment speeds. Petrova et al. (2019) utilized fluorescence microscopy to visualize solid-to-solid contact regimes. As it is a very sensitive observation method, the fluorescence technique is also used more frequently in other fields 
of studies, such as chemistry (Rozaini et al., 2012), biology (Hötzer et al., 2012), and medicine (Marcu, 2012).

As the contact condition of tires is closely related to the coefficient of friction and the grid performance of tires, a lot of data is required to design and produce better performing tires. The information about the contact condition of tires can also be used to analyze the condition of tire wear, to predict the lifetime of tires, and for some other purposes. However, the currently existing reports and data are still far from enough to satisfy the needs of data on many circumstances of tire contact conditions. One of them is the contact condition of rubber tires due to the influence of moving speeds and applied loads on wet surfaces.

Therefore, to contribute to providing data for these purposes, this study conducted an experiment to investigate how the applied loads and moving speeds affect the contact condition of rubber tires. As a fundamental study, the experiment was carried out as a rubber tire wheel running on the top of the flat surface of a glass plate under several applied loads and moving speeds. The contact condition of the tire and mating surface was observed using the ultraviolet-induced fluorescence technique. The moving speed in this study indicates free rotation, which is a mixed movement of rolling and sliding. The rubber wheel had the same material as the tire rubber of regular vehicles, and the mating surface was a smooth glass plate (BK7). During the experiment, the fluorescence liquid made of the pyranine-dyed solution was covered on the top of the mating surface, simulating the condition of wet surfaces.

\section{Methods}

\subsection{Experimental Setup}

The experiment of measuring the contact area was operated using a smooth surface of a cylindrical rubber specimen and a smooth surface glass plate. During the test, the glass plate surface was covered by pyranine solution representing wet conditions in the rainy season. As shown in Figure 1, the rubber specimen was shaped in the form of a cylinder with an outer diameter, an inner diameter, and a width of $80 \mathrm{~mm}, 42 \mathrm{~mm}$, and $32 \mathrm{~mm}$, respectively.

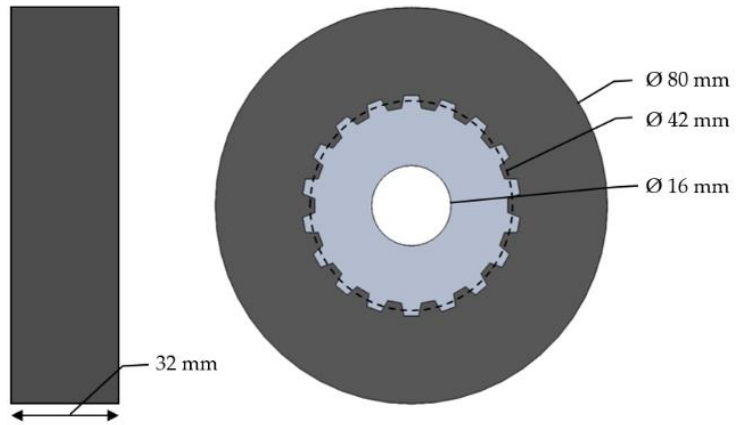

Figure 1 Rubber-wheel dimensions

A gear-patterned aluminum was added to the inner side of the rubber specimen with a hole diameter of $16 \mathrm{~mm}$. It helped the rubber specimen to connect to the linear guide for the dynamic contact experiment. This dimension is a commonly used rubber size for laboratory-scale experiments, referring to ISO 23233 in LAT 100 (Laboratory Abrasion Tester 100). The diameter of the rubber specimen was similar; however, the thickness was twice larger due to the supplier's (TOYO TIRE corporation, a tire manufacturer company in Japan) reason. The mating contact was a glass plate branded as BK7 in a rectangular shape 
with a width of $100 \mathrm{~mm}$ and a thickness of $5 \mathrm{~mm}$. The rubber specimen was connected to a linear guide device through a shaft with the possibility of normal loading adjustment. The details of the flat surface of the glass plate and rubber specimen materials are shown in Tables 1 and 2, respectively.

Table 1 Specification of glass plate

\begin{tabular}{lc}
\hline \multicolumn{1}{c}{ Specimen } & BK7 \\
\hline Dimensions $(\mathrm{mm})$ & $100 \times 100 \times 5$ \\
Density $\left(\mathrm{g} / \mathrm{cm}^{3}\right)$ & 2.51 \\
Poisson's ratio & 0.206 \\
Young's modulus (MPa) & $82 \times 10^{3}$ \\
Refractive index & $1.53627(365 \mathrm{~nm})$ \\
Internal transmittance & $0.988(365 \mathrm{~nm})$ \\
\hline
\end{tabular}

Table 2 Specification of rubber-wheel material

\begin{tabular}{lc}
\hline \multicolumn{1}{c}{ Specimen } & Tire rubber \\
\hline Diameter (mm) & 80 \\
Width (mm) & 32 \\
Poisson's ratio & 0.49 \\
Young's modulus (MPa) & 2 \\
\hline
\end{tabular}

The observation of the rubber specimen contact area was provided by fluorescence microscopy using ultraviolet light as an excitation light source and pyranine compound as a fluorescent dye. The ultraviolet light source was generated by a ring-shaped ultraviolet device with a $365 \mathrm{~nm}$ peak of wavelength. It was situated below the mating surface and above a high-speed camera. Also, a dichroic filter with a passing wavelength of between 505 $\mathrm{nm}$ and $575 \mathrm{~nm}$ was placed on the top of the camera to avoid other lights than the green color fluorescence light for a more accurate analysis. This arrangement allowed us to directly observe the contact condition of rubber specimens on a flat surface. Finally, the image of the rubber-wheel contact area was captured and recorded using a high-speed digital camera, HAS-U2 (manufactured by DITECT Ltd. of Japan). The outline of fluorescence microscopy used in the current study is shown in Figure 2.

The pyranine substance used as a fluorescence dye in microscopy has a chemical composition of $\mathrm{C}_{16} \mathrm{H}_{7} \mathrm{Na}_{3} \mathrm{O}_{10} \mathrm{~S}_{3}$ with a molecular weight of $524.39 \mathrm{~g} / \mathrm{mol}$. The pyranine dye was adopted in this study due to its advantage of having a wide-range stock shift. It enables the researchers to distinguish the contact and noncontact regions without the interference of the excitation light source. The pyranine solution was made by mixing the pyranine substance with distilled water according to the desired density (3200 mg/L). In the process of fluorescence, the pyranine solution absorbs the excitation light, and then it instantly emits green fluorescence light with a peak wavelength of $513 \mathrm{~nm}$. In addition, to avoid external factors' influences on the fluorescence intensity such as temperature and humidity, the experiment was conducted in a room with identical temperature and humidity of $22.1^{\circ} \mathrm{C}$ and $54 \%$, respectively. 


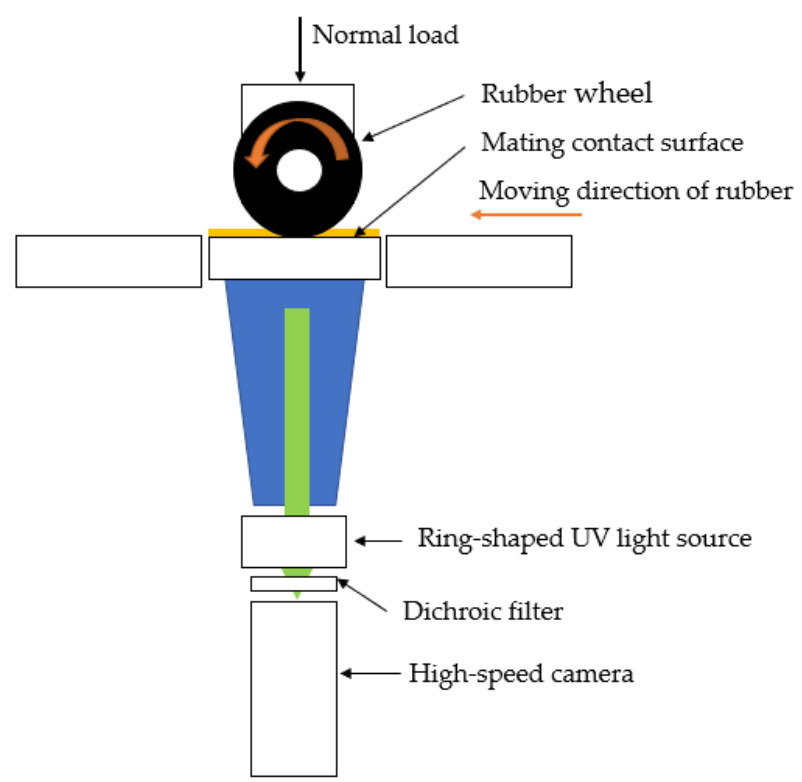

Figure 2 The schematic figure of fluorescence microscopy setup

\subsection{Condition and Procedure}

The experiment was carried out under stationary (applied-load dependency) and dynamic (moving-speed dependency) conditions. In the stationary state, the weight placed on the experiment apparatus ranged from $40.2 \mathrm{~N}$ to $157.7 \mathrm{~N}$ with nine selected variations of applied loads. To solve the problems of creep deformation that make the rubber material keep deforming over the pressing time, a creep deformation test was conducted. The result showed that the pressing time was similar to the rubber contact on a rough surface conducted in the previous experiment, which was 300 s (Rahman et al., 2020). Consequently, the rubber specimen contact area due to applied-load dependency was captured $300 \mathrm{~s}$ after the rubber specimen bumped into the flat surface. To ensure that the rubber specimen returned to its initial state after the test, the rubber specimen had to wait for about $300 \mathrm{~s}$ before conducting the next applied-load test. As for moving rubber contact, the experiment was carried out with several moving speeds and applied loads: the moving speed ranged from $4 \mathrm{~mm} / \mathrm{s}$ to $160 \mathrm{~mm} / \mathrm{s}$ with six variations and three different applied loads of $60.8 \mathrm{~N}, 80.4 \mathrm{~N}$, and $99 \mathrm{~N}$. Detailed information about the experimental conditions under static and dynamic conditions is shown in Table 3. The moving rubber specimen in the current study performed free rotation with mixed movements of rolling and sliding. The ratio of rolling and sliding in the moving rubber specimen was generated naturally based on the moving speed.

Table 3 Experimental conditions on moving rubber specimen contact

\begin{tabular}{lcc}
\hline \multicolumn{1}{c}{ Contact condition } & Applied load $(\mathrm{N})$ & Moving speed $(\mathrm{mm} / \mathrm{s})$ \\
\hline Static contact & $40.2-157.7$ & 0 \\
Dynamic contact & $60.8,80.4,99$ & $4-160$ \\
\hline
\end{tabular}

The contact area of the rubber specimen was captured using a high-speed camera. Following that, the image of rubber specimen contact was processed using image processing software known as Image-J to measure the contact area between the rubber specimen and flat surface. In this study, the measured contact area was the apparent contact area. The boundary between the apparent contact area and noncontact region was determined based on a series formula developed by Nobuyuki Otsu in 1979, a well-known 
expert in image segmentation analysis (Otsu, 1979). This method is known as the Otsu thresholding method. The boundary of apparent contact area calculated using the Otsu thresholding method was at the intensity of 36 . Thus, the intensity of 36 and below was recognized as the apparent contact area, and the intensity above 36 was categorized as the noncontact region. Figure 3 shows the flow chart of the experimental procedure using fluorescence microscopy to measure the rubber specimen contact area.

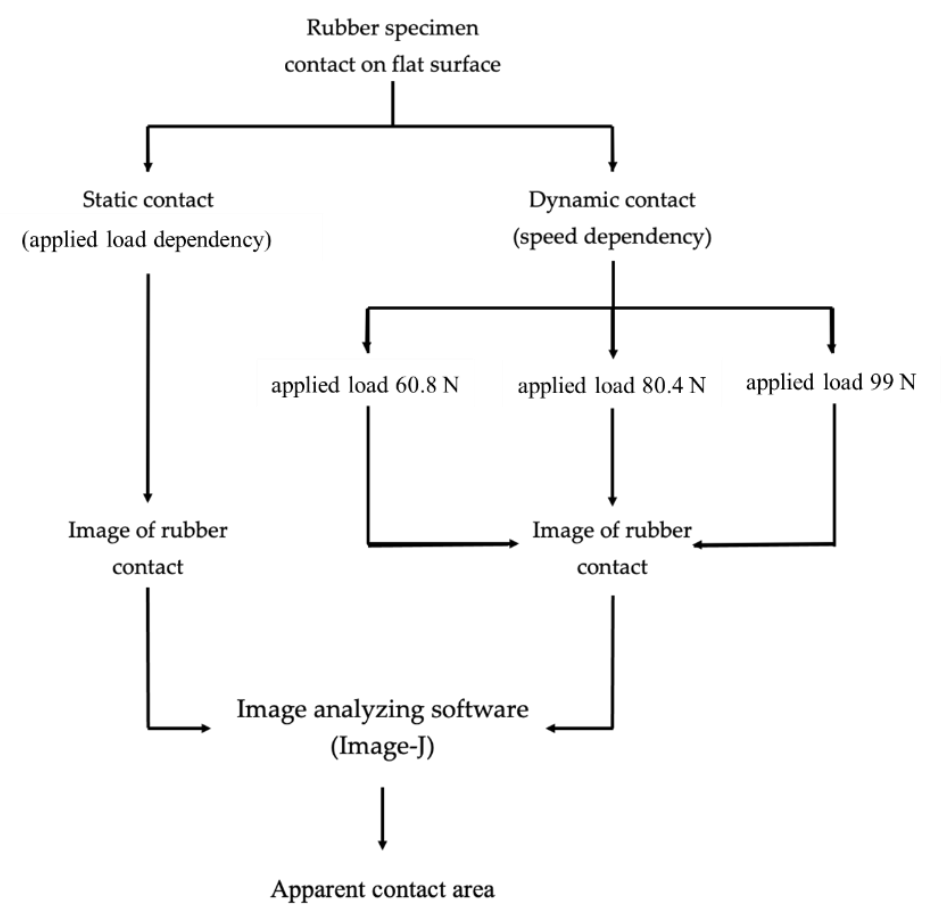

Figure 3 The flow chart of the experiment

\section{Results and Discussion}

\subsection{Applied-Load Dependency}

As the rubber specimen was in contact with a flat surface, the main cause of the coefficient of friction was the adhesion force between the rubber specimen and the glass plate. At the same time, the applied load on the rubber wheel widened the range of contact area due to its viscoelasticity. Figure 4 shows the binary image of the apparent contact area between the rubber wheel and the flat surface for nine selected applied loads. The experiment was executed three times to obtain more accurate data in applied-load dependency.

As shown in Figure 4, the apparent contact area between the rubber wheel specimen and the flat glass plate was wider as the applied load increased, indicating load influences on rubber wheel specimen contact. The higher the load applied on the rubber wheel specimen, the more it deformed and then formed a wider contact area. Previously, we reported the load dependency of rubber specimen contact in a conference (Rahman et al., 2018). However, we could not identify the increasing pattern of the contact area because the amount of data was insufficient. Therefore, in the current study, we conducted experiments with enough variation of applied loads to obtain more precise information. Figure 5 shows the graph of the plotted apparent contact area of the rubber wheel specimen based on applied-load dependency in the log-log axis. The plotted measured apparent contact areas of the rubber wheel specimen for every applied load from three tests were distributed within the range of approximately $20 \mathrm{~mm}^{2}$ on each applied load. Also, the 
apparent contact area of the rubber specimen in the graph with the log-log axis increased linearly as the applied load increased. The log-log axis is usually used by researchers who study contact mechanics to describe whether the obtained data is following the theoretical formula in contact mechanics or not. As shown in Figure 5, a straight trend line was formed. This means that the obtained results agreed with the predicted contact theory of Hertz, as the half-width of the contact area is proportional to the applied load W1/2 (Johnson, 1987).

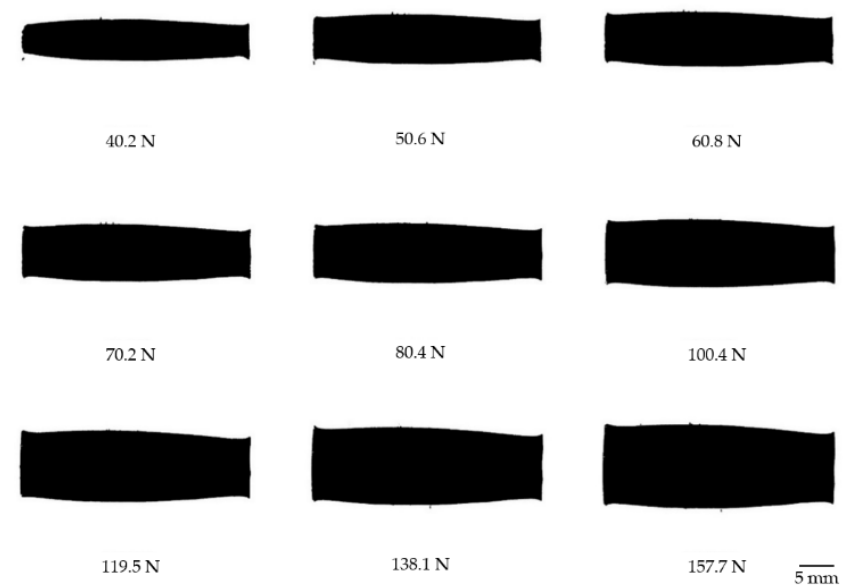

Figure 4 Images of rubber specimen apparent contact area due to various applied loads (the images belong to trial 2)

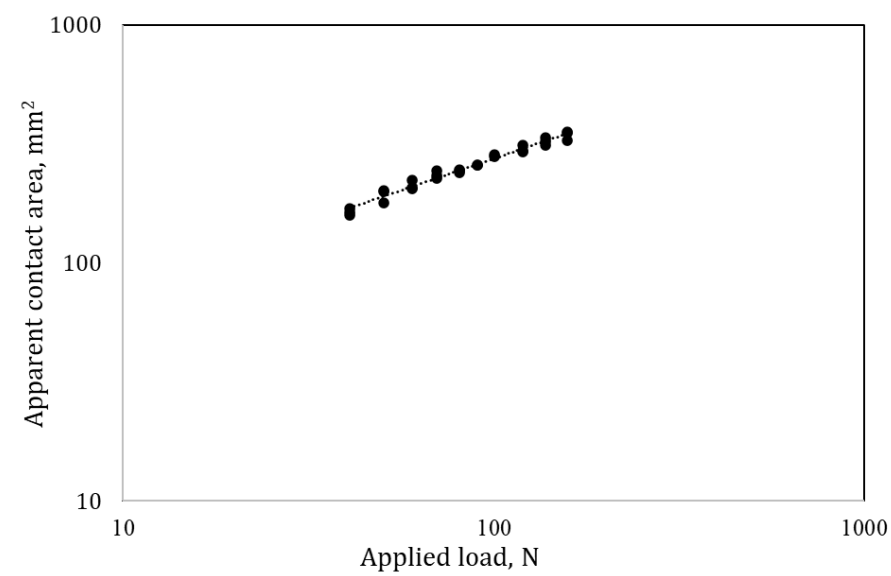

Figure 5 Rubber specimen apparent contact area vs. applied load

\subsection{Moving-Speed Influence}

Figure 6 displays the binary images of rubber specimen contact due to variant moving speeds ranging from $4 \mathrm{~mm} / \mathrm{s}$ to $160 \mathrm{~mm} / \mathrm{s}$. Something that popped out in the images of rubber wheel specimen contact was air bubbles, which sometimes attached to the surface of the glass plate and moved along with the rubber wheel specimen forming air pillars. The interference of air pillars was omitted from the calculation of the contact area of the rubber wheel specimen. Figure 7 shows the graph of the rubber wheel specimen apparent contact area due to the influence of six selected moving speeds under three chosen applied loads. Overall, the apparent contact area of the rubber specimen fluctuated as it increased, reaching the highest at a moving speed of $8 \mathrm{~mm} / \mathrm{s}$, and then decreased gradually as the moving speed increased. The graph also shows the effect of the applied load on the moving rubber specimen, as the apparent contact area increased along with the applied loads. 


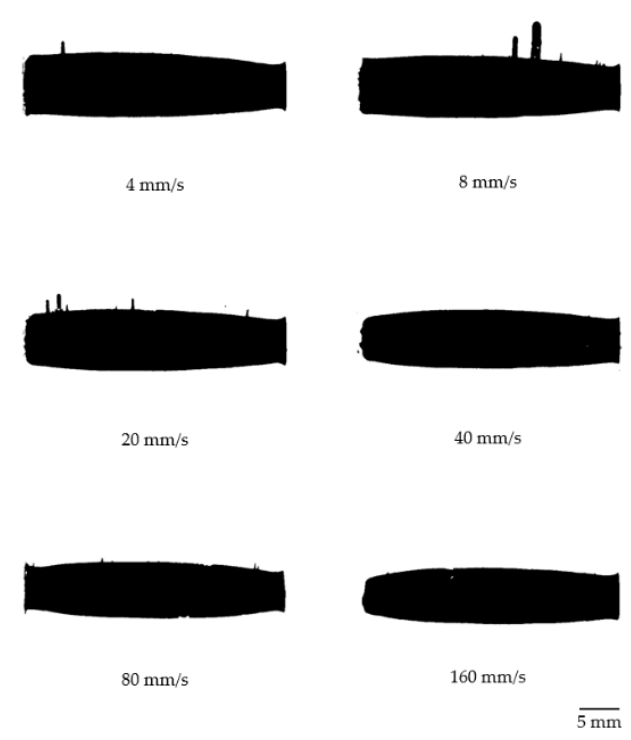

Figure 6 Rubber specimen contact area due to the influence of moving speed (applied load at 80.4 N)

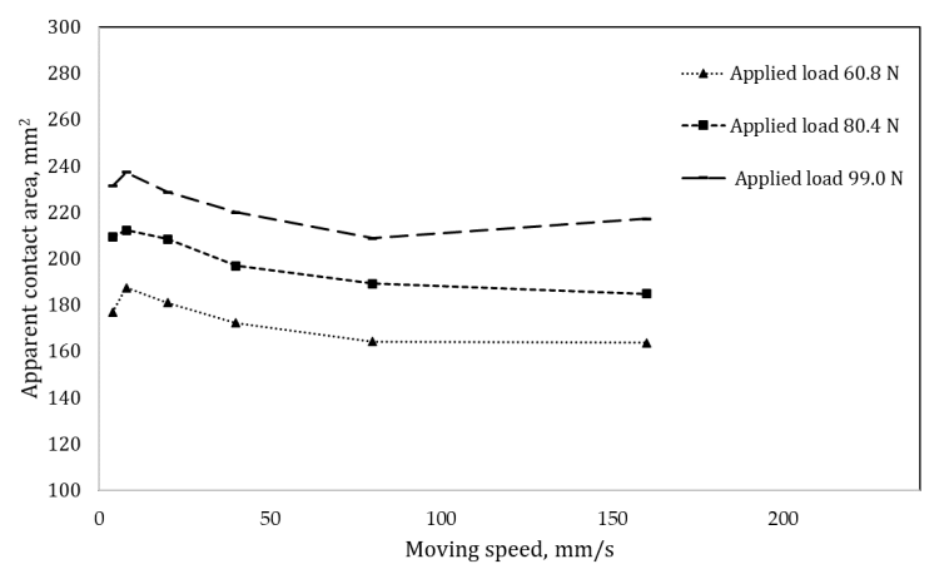

Figure 7 Rubber specimen apparent contact area on dynamic condition with three variants of applied load: $60.8 \mathrm{~N}, 80.4 \mathrm{~N}$, and $99 \mathrm{~N}$

As reported by Michelin (2001), the rubber material is divided into three states based on the influence of stress frequency. The first is a rubbery state that occurs at low frequency. Next, as the frequency increases, the rubber material appears to be viscoelastic, which is the ideal condition for grip performance. Lastly, it becomes a glassy state as the frequency increases further. Moreover, the adhesion bond between the rubber specimen and flat glass surface is formed and separated repeatedly as the rubber specimen moves along the path. Based on these conditions, at a lower speed where the stress frequency of the rubber is low, the apparent contact area of the rubber specimen increased as speed increased. The possible reason is likely due to similar phenomena of junction growth theory developed by Tabor (1960). When the rubber specimen was moving at a lower speed, due to strong adhesion force, the separation speed between rubber and flat surfaces was lower than that of the formation speed, making it look like the junction between the rubber wheel and flat surface growth. As a result, the apparent contact area increased as the moving speed increased up to $8 \mathrm{~mm} / \mathrm{s}$. This result has a tendency similar to the experimental result (in the form of coefficient of friction vs. speed) and the theoretical approach developed by Klueppel et al. (2003). However, as the moving speed increased to more than $8 \mathrm{~mm} / \mathrm{s}$, the 
viscous characteristic of rubber became dominant, which led to a glassy state. Therefore, the apparent contact area of the rubber specimen decreased as the moving speed increased.

\section{Conclusions}

The effect of the applied load and moving speed on the free rotating rubber specimen contact area on a smooth-surfaced glass plate was studied. The results indicate different tendencies between applied-load and moving-speed dependencies. Regarding applied-load dependency, the plot distribution of the rubber specimen apparent contact area was within the $20 \mathrm{~mm}^{2}$ range for each applied load from three tests. Overall, the apparent contact area increased linearly as the applied load increased in the graph with the log-log axis. This result fits the function of the Hertz theory, as the half-width of the contact area is proportional to the applied load in the power of $1 / 2$. Regarding moving-speed influences, some information can be obtained from the results. First, the applied load also increased the apparent contact area of the rubber specimen. Second, the moving speed of the rubber specimen increased the rubber apparent contact area at a lower speed up to $8 \mathrm{~mm} / \mathrm{s}$, and then it decreased as the moving speed increased further.

\section{Acknowledgements}

This work was supported by the Indonesia Endowment Fund for Education (LPDP), the Ministry of Finance of Republic Indonesia (KEMENKEU), the Ministry of Research and Technology of Republic Indonesia (KEMENRISTEK), and the Ministry of Education and Culture of Republic Indonesia (KEMENDIKBUD) in the form of a scholarship for a doctoral degree at Kanazawa University with the grand contract number PJR-1570/LPDP.4/2019. Also, thanks to TOYO TIRE for the financial support and being a collaboration partner with the Tribology Laboratory of Kanazawa University.

\section{References}

Bódai, G., Goda, T.J., 2012. Friction Force Measurement at Windscreen Wiper/Glass Contact. Tribology Letters, Volume 45(3), pp. 515-523

Fowell, M.T., Myant, C., Spikes, H.A., Kadiric, A., 2014. A Study of Lubricant Film Thickness in Compliant Contacts of Elastomeric Seal Materials using a Laser Induced Fluorescence Technique. Tribology International, Volume 80, pp. 76-89

Fujii, Y., 2008. Method for Measuring Transient Friction Coefficients for Rubber Wiper Blades on Glass Surface. Tribology International, Volume 41(1), pp. 17-23

Hasan, A., Aznury, M., Purnamasari, I., Manawan, M., Liza, C., 2020. Curing Characteristics and Physical Properties of Natural Rubber Composites using Modified Clay Filler. International Journal of Technology, Volume 11(4), pp. 830-841

Hötzer, B., Medintz, I.L., Hildebrandt, N., 2012. Fluorescence in Nanobiotechnology: Sophisticated Fluorophores for Novel Applications. Small, Volume 8(15), pp. 22972326

Johnson, K.L., 1987. Contact Mechanics. Cambridge, United Kingdom: Cambridge University Press

Klueppel, M., Muller, A., Le Gal, A., Heinrich, G., 2003. Dynamic Contact of Tires with Road Tracks. In: 2003 Technical Meeting of the American Chemical Society, Rubber Division, San Francisco, USA, Paper No. 49

Liang, C., Li, H., Mousavi, H., Wang, G., Yu, K., 2020. Evaluation and Improvement of Tire Rolling Resistance and Grip Performance based on Test and Simulation. Advances in Mechanical Engineering, Volume 12(12), pp. 1-14 
Maegawa, S., Itoigawa, F., Nakamura, T., 2015. Optical Measurements of Real Contact Area and Tangential Contact Stiffness in Rough Contact Interface Between an Adhesive Soft Elastomer and a Glass Plate. Journal of Advanced Mechanical Design, Systems, and Manufacturing, Volume 9(5), pp. 1-14

Marcu, L., 2012. Fluorescence Lifetime Techniques in Medical Applications. Annals of Biomedical Engineering, Volume 40(2), pp. 304-331

Michelin, 2001. The Tyre: Grip. Clermont-Ferrand, France: Société de Technologie Michelin

Nakajima, Y., Takahashi, F., 2002. Increase of Frictional Force of Rubber Block by Uniform Contact Pressure Distribution and Its Application to Tire. Rubber Chemistry and Technology, Volume 75(4), pp. 589-604

Otsu, N., 1979. A Threshold Selection Method from Gray-Level Histograms. IEEE Transactions on Systems, Man, and Cybernetics, Volume 9(1), pp. 62-66

Persson, P., 2001. Theory of Rubber Friction and Contact Mechanics. The Journal of Chemical Physics, Volume 115(8), pp. 3840-3861

Persson, P., Volokitin, A., 2006. Rubber Friction on Smooth Surfaces. The European Physical Journal E, Volume 21(1), pp. 69-80

Petrova, D., Weber, B., Allain, C., Audebert, P., Venner, C.H., Brouwer, A.M., Bonn, D., 2019. Fluorescence Microscopy Visualization of the Roughness-Induced Transition Between Lubrication Regimes. Science Advances, Volume 5(12), pp. 1-8

Rahman, J., Iwai, T., Koshiba, K., Shoukaku, Y., 2018. Observation of Contact Area of Rubber Wheel using an Ultraviolet-Induced Fluorescence Method. In: Proceedings of Asia International Conference on Tribology 2018, Serawak, 17-20 September, Malaysia, pp. 291-292

Rahman, J., Shoukaku, Y., Iwai, T., 2020. In Situ Rubber-Wheel Contact on Road Surface using Ultraviolet-Induced Fluorescence Method. Applied Sciences, Volume 10(24), pp. 1-13

Rozaini, M., Ali, R., Ros, L., 2012. Normal Micellar Value Determination in Singular and Mixed Surfactant System Employing Fluorescence Technique. International Journal of Technology, Volume 3(4), pp. 103-109

Tabor, D., 1960. Hysteresis Losses in the Friction of Lubricated Rubber. Rubber Chemistry and Technology, Volume 33(1), pp. 142-150

Zheng, B., Huang, X., Zhang, W., Zhao, R., Zhu, S., 2018. Adhesion Characteristics of TireAsphalt Pavement Interface based on a Proposed Tire Hydroplaning Model. Advances in Material Science and Engineering, Volume 2018, pp. 1-12 